\title{
'It's all bad news': the first 3 months following a diagnosis of malignant pleural mesothelioma
}

Anne Arber, School of Health and Social Care, University of Surrey, Guildford, UK Lesley Spencer, The Princess Alice Hospice, West End Lane, Esher, Surrey UK

\begin{abstract}
Objective: This study explores patient's experience during the first three months following a diagnosis of malignant pleural mesothelioma.

Methods: This study uses a grounded theory approach with semi-structured, face-toface interviews with ten patients during the first 3 months following diagnosis.

Results: The key concept that emerged from the data was this: Uncertainty and lack of control, leading to emotional, physical and psychosocial distress. Three themes informed the key concept: 'it's all bad news', 'good and bad days' and strategies of amelioration. Patients worked with a short-term perspective on their illness but worried about the long-term outcome and the speed of their deterioration.

Conclusion: Patients receive insufficient psychosocial support during the first three months following diagnosis. An early palliative care referral would improve support and referral strategies during the first three months of the disease.
\end{abstract}

\section{Introduction}

Malignant pleural mesothelioma (MPM) is a complex and difficult cancer affecting the lining of the lung. MPM is linked to exposure to asbestos and invariably leads to a short life expectancy [1]. However there is reported to be a huge variability in the course of the illness with some patients experiencing a slower disease progression and others more aggressive disease [2]. MPM is more likely to occur in late middle age with a long latency period between exposure to asbestos and the appearance of the disease [3]. There can be problems in achieving a diagnosis in the early stages and this is because symptoms such as chest pain can be mistaken for cardiac disease [4,5]; alongside this are quality of life issues related to emotional distress, experience of symptom such as pain and breathlessness and complicated medicolegal and financial concerns $[4,5]$. So far treatment has not achieved improvement in survival and palliative and supportive care is important to sustaining a good quality of life [6,7]. Research on this patient group is limited and care and support is based on the needs and care pathways of those with lung cancer, which may not reflect the concerns of those with MPM [8]. With the incidence of MPM rising and expected to peak between 2010 and 2015, it is timely to produce research evidence related to the 
patient's experience in the early phase of the illness following diagnosis and to investigate how patients are supported. This study is concerned with the patient's experience in the first three months following diagnosis of malignant pleural mesothelioma (MPM).

\section{Literature Review}

Malignant Pleural Mesothelioma is the most common cancer of the pleural cavity and $80 \%$ of mesothelioma's originate in the pleural space [9]. The typical presentation is chest pain and dyspnoea [4,5]. The pain may involve a heavy feeling in the chest, aching in the shoulder and arm, chest wall and upper abdomen. The disease is invariably fatal, although there are exceptions [8 ] with some experiencing periods of stability. However many people die within a year of diagnosis [1].

The care of people with mesothelioma in Australia has been described as a 'medical lottery' as patients may not receive the referrals and services they need [7]. Patients and carers of those with MPM report many unmet needs with $90 \%$ of patients reporting pain and breathlessness, cough, appetite loss, fatigue, sleep disturbance and sweating $[8,9]$ most significantly psychosocial distress is reported as a serious issue for patients and families [4,5,10]. Sweeney (a medical doctor) describes severe emotional distress following his diagnosis of MPM experiencing inadequate support from healthcare staff who avoided his distress [11]. The diagnosis is difficult for health care practitioners to deliver because of the lack of effective treatments and the short prognosis.

Charmaz (1997) reports the catastrophic loss of control over life experienced by those living with the consequences of chronic illness and advanced cancer with the loss of self-esteem resulting in a diminished self $[11,12]$. In studies of patients with mesothelioma it is reported that they felt their health was controlled by external factors such as luck or powerful others and this resulted in depression, anxiety, helplessness and fatalistic attitudes $[13,14]$. The cultural imperative to think positively about cancer may mean that those who have reached a point where this is not possible may find themselves alone in confronting their mortality $[15,16]$. Charmaz [17] observes how losses "are most marked at the onset of a serious, debilitating illness or at points when ill persons define former actions, lives and selves as now precluded by illness" [17].

In a study of patients with small cell lung cancer it was found that doctors rarely discussed the course of the illness with patients [18]. Specialists tended to focus on the short-term aspects of illness such as treatment schedules, laboratory tests, 
investigations and generally did not discuss the longer-term aspects of the illness $[19,20]$. The advantage of focusing on the short-term perspective is that it focuses attention on treatment and instils optimism around treatment schedules and avoids the poor outcomes. The short-term perspective provides structure to the illness experience and helps the patient (and the staff) with this difficult period by making it orderly and manageable [19]. Although there is considerable research into the experience of lung cancer, little research has focused on the early phase of the illness following diagnosis of MPM and the short-term and longer-term concerns from the patient's perspective.

\section{Aim}

To explore patient's experience during the first 3 months following a diagnosis of MPM.

\section{Method}

This study used a grounded theory approach [21], where theoretical propositions and theories are 'grounded' in the reality of the data collected [22]. This approach was used to discover what was going on in relation to the participant's recently diagnosed with pleural mesothelioma. Theoretical sampling was used where data were collected, coded and analysed and then decisions were made about where next to find data; this process was repeated until data saturation was achieved with ten participants and included two women [21,23]. The sampling strategy was affected by the difficulty in recruiting patients due to the small numbers of patients on two hospital sites. Two patients were too ill to be interviewed and it took one year to recruit 10 people to the study. Eventually, ten patients (eight men and two women) were recruited from two acute trusts in the South of England (See Table 1 for patient characteristics and performance status). All participants apart from one (John) were interviewed in their home, with John being interviewed in the out-patient department of the local hospital.

Data were collected by open-ended, audio-recorded interviews where participants were asked open ended questions about their experience leading up to diagnosis, receiving the news of their illness and subsequent experiences of treatment, care 
and coping. One participant (John) did not want the interview audio-recorded but he agreed for the researcher to take notes. Later on in the interviews more closed ended questions were asked to clarify points about symptom experience and support without distorting the rapport built up with participants by use of open-ended questions. The function of interviews in grounded theory is to draw out the participant's story. For example the researcher started by asking: 'When did you first notice something was wrong?' Probing questions were used to encourage the participants to tell their story of their experience and to ensure that the researcher fully understood the meaning of what was being said as well as the context. During the first interview the issue of uncertainty was raised: 'It's just the uncertainty really'. This became an important theoretical construct and the work of Charmaz [12] related to chronic illness was used to explore and develop this concept further [12]. Using the constant comparative method the issues of uncertainty and 'bad news' were found to be relevant in all subsequent interviews. The constant comparative method was used for analysis of the transcribed data with text coded and compared for similarities and differences until substantive categories and themes emerged. The interview transcripts were coded line by line and key words such as 'uncertainty' 'bad news' were highlighted. Initial patterns began to emerge from the first interview, and this informed further interviews. The key words and phases were highlighted on the transcript, categories were formed that supported the key concept of uncertainty and loss of control. Emerging codes were compared to existing codes, to examine similarities or differences. As data analysis progressed, relationships between the categories and subcategories were developed through axial coding [24]. Analysis continued until each category was refined and saturated and no new properties emerged [24]. The research proposal was approved by the National Research Ethics Service, the Hospital Trust's Research and Development Committee and the University Ethics Committee. Recruitment to the study took place during 2008-2009 and one researcher carried out all the interviews.

\section{Findings}

The key concept that emerged from the data was: uncertainty and lack of control, which lead to emotional, physical and psychosocial distress during the first three months following diagnosis. Three themes informed the key concept: 'it's all bad news', 'good days and bad days' and strategies of amelioration. 


\section{Uncertainty and lack of control}

All participants report high levels of uncertainty and feelings of a lack of control leading to psychosocial distress since receiving their diagnosis. In mesothelioma unpredictability is linked to uncertain disease trajectory and the poor prognosis [3]. Participants describe the shock of receiving the diagnosis and the uncertainties related to how the disease will progress and the timescale involved in their physical decline.

Ivan was preparing for the worst case scenario thinking that he was going to die very soon. He was planning and preparing for his death and his funeral. Ivan goes on to talk about his uncertainties and struggles in relation to his life expectancy:

I don't know what tomorrow will bring, or next month, I don't even know if I can look to Christmas....really, I don't know the speed of these things or...but I just don't know. Something might turn up, who knows.

Ivan reports great anxieties around the speed of his disease progression and how long he can expect to live. He uses 'I don't know' four times in this data extract emphasising his loss of certainty in the future whether that is tomorrow or at another date in the future. In particular he is worried about the speed of his decline and what is going to happen to him. He can only think that something might turn up. He is in despair about the loss of certainty in his life and there is a felling that nobody can help him with this as 'who knows'. There is a feeling from Ivan that no one is in control of this situation.

Uncertainty in cancer has been called the 'Damocles Syndrome' not knowing when the tragedy will occur but knowing it is unavoidable leads to distress and suffering [25]. Uncertainty is most acute during the early stages of illness following diagnosis [26]. Participants also face worries about the treatment schedule, Henry, describes the uncertainties he faces in the medical system. He describes how he has been in 'limbo' following his radiotherapy:

I had a session at [Cancer Centre]. There is no liaison. I finished me radiotherapy but then I was left in limbo... There doesn't seem to be a link with the patient... I mean I got home and I thought well what happens now ${ }_{2}$ you know. 
Henry is confused about what is going to happen next in the treatment trajectory. He lacks information and understanding about the next step in the treatment trajectory leading to feelings of uncertainty and distress around 'what happens now'.

John describes concerns and worries for his wife who washed his clothes, which were covered in asbestos when he was working for a heating company:

Hoping it is not going to contaminate my wife she washed my clothes when I was working for the heating company and I had asbestos all over them.

John uses the word 'contaminate' in this sense his wife is also in danger and potentially 'contaminated' because of the asbestos being transferred from his clothes to his home. John came across as very angry and anxious during the interview. He was the only participant to request the interview to be held outside of the home and did not want it to be audio-recorded; however he agreed to the researcher taking written notes.

John is also are working closely with solicitors who visit him at home. He is awaiting the outcome of investigations in relation to the possibility of making a claim against two previous employers regarding his contact with asbestos during his work as a heating engineer:

The only thing I'm waiting for is the solicitor following those two companies, which are no longer trading. I have to wait and see and not sure of the outcome.

The feelings of distress and despair communicated during all the interviews were related to loss of control not only of the body but of one's life. The speed at which things were happening led to an incomprehension about what was going to happen next and support the findings of Charmaz [12] around the loss of control over life [12]. The language of distress and uncertainty used by participants were communicated using words such as: 'not sure', 'I'm waiting', 'contaminate' , 'left in limbo', 'what happens now?', 'I don't know', 'who knows'. These words reflect the loss of control and insecurity about how to live and who can possibly help.

The following sub themes support the main themes and are the components of uncertainty, and loss of control of life leading to distress but tempered by strategies of amelioration. 


\section{'It's all bad news'}

All the participants found it difficult to cope with their diagnosis due to all the negative information and 'bad news' around MPM and this led to feelings of depair.

Henry says: it's all bad news isn't it?

By this question there is an expectation that the interviewer also shares the perspective on the bad news of having mesothelioma.

Chris while acknowledging the bad news doesn't want to think about or dwell on the future therefore he prefers to bury his head in the sand:

It doesn't pay to think too far down the road because that's bad news. As you say, head in the sand.

The metaphor of 'head in the sand' is one way to deal with a devastating prognosis and this was a mechanism of keeping the bad news at bay for now. This helped Chris to avoid the realisation of the 'bad news' and what the future holds by avoiding thinking about it. Denial has been found to be a common psychological response in those with mesothelioma and industrially acquired lung disease $[13,14]$.

Doug also feels that all the information he has received is negative:

...negative information around having such a disease.

The feeling of negativity around the diagnosis is difficult for participants to come to terms with and it immediately fills people with dread about the future and what the illness means.

The poor prognosis related to MPM causes a dilemma in relation to whether to continue treatment given the poor outlook presented by medical staff as well as the negative information in patient information booklets and on the Internet. For example Anita, who had been investigated for around 12 months for a cardiac illness before being diagnosed described how she started a course of chemotherapy but felt that it was compromising her quality of life due to all the side effects she was having:

I've just been on the Internet site for meso and ahm in the book that I had they gave me on it, it said there wasn't much point in having chemotherapy you know it ahm. So when I was so ill after that first session I was really ill I just couldn't face going having it all done again.

Anita took the decision to discontinue chemotherapy treatment and then sought a referral to the palliative care team. The negative attitude to the disease appears to be communicated to patients in a number of ways both in the written material offered 
to patients in information booklets, in the verbal and non-verbal behaviour of staff [11] and from material on the Internet. Clayson's study participants also report being given a hopeless message by medical staff who were reported to have said : 'I can't give you much hope', and another, 'we don't think we can help you'; and another, 'she said it's mesothelioma, it's malignant, it's terminal and there's no treatment' [4].

\section{'Good days and bad days'}

Uncertainties about daily living in serious illness when good and bad days occur restricts peoples' lives [11]. Leading a restricted life means avoidance of physical activity, and reduced social contacts, which protects the person but at a cost to their self-image.

Doug describes 'good days and bad days':

...good days and bad days' and 'if I have a late night l'm affected the following day".

Participants experienced tiredness and fatigue which is a common problem in MPM [9]. None of the participants were breathless at rest, during the interview but described some breathlessness on exertion. Breathlessness for many participants improved following medical pleurodesis to treat pleural effusion.

Those that reported pain describe mild pain such as an ache, tenderness or soreness in the chest. John describes a little ache:

I've noticed a little ache in the chest (rubbing his chest) but doesn't affect me sleeping

Anita has an ache in her side that is tender and felt a pressure in her chest:

It feels as though there is just something pressing down on you all the time.

Gill describes pain that is not controlled with Paracetamol. She has now been prescribed Tramadol to help with her pain:

The centre of the chest is painful but it's only sore to touch, but I do get pain but the pain is mostly at night, I think during the day, because you, you are talking and you are pottering about, you take no notice, it isn't pain that's there continually.

Patients are experiencing pain but not necessarily reporting it to hospital staff. Gill needed stronger analgesics for her pain and there was a delay in getting Tramadol. 
The fact that pain was not reported as a major problem early on in the disease is found in other studies [4]. However patients did describe aches, soreness and tenderness around the chest but were more likely to describe discomfort rather than pain which was managed by analgesics.

Participant's report feeling distressed when experiencing sweating particularly at night:

Doug: And the other thing too that I've noticed and it's causing arguments between us, is that it's almost like what I imagine the female menopause to be. I'm sweating and I'm hot.

Interviewer: Is that any time or at night?

Doug: No it's, if it gets warm or the heating's on or I'm, we went shopping to a big shop, Macros, last week with two friends, and I had to go out and sit in the car. They were laughing at me because l'd taken me coat off and they said they were cold, but I was sort of sweating, until I got outside, got in the colder air, sat in the car, opened all the windows and I was all right, just sitting there and it had gone.

Sweating is a difficult problem for Doug as his friends are laughing at him and his wife is also finding it difficult to cope with. Henry also reports night sweats that he describes as 'horrible' and this disturbs his sleep:
Henry: $\quad$ Yeah the sweating yeah...
Interviewer:: and is that every night or?
Henry: $\quad$ Three times a night I have to change.

The day and night sweats caused a lot of distress to patients. In particular it disturbed sleep at night and the feeling of coldness was also a persistent problem for some people. In a case note review of 80 patients with mesothelioma, sweating was reported in $14(18 \%)$ of the case notes studied [4]. In the present study half of the participants experienced sweating.

\section{Strategies of amelioration:}

Participants did find positive events that helped them cope with the illness. Charmaz [12] calls finding positive events as a turning point because it 'demonstrates resiliency of self' and it presents the individual as having taken control of the illness rather than the illness taking control of the person [12]. Participants describe a number of strategies to help them cope such as use of complimentary and alternative medicine (CAM), visits from the Asbestos Support and Awareness Group (ASAG), and referral to palliative care. 
Ivan is using CAM involving a special diet and this provided Ivan with a feeling of control and that he could do something positive to help himself and helped him cope with the many uncertainties around his illness.

Many of the patients on one of the study sites received excellent support from the Asbestos Support Group (ASAG) within their locality:

I've had help from ASAG, wonderful people... She filled in the forms for the disability. I haven't had to do anything (John)

Participants had received home visits from legal representatives and from members of ASAG, which is a charity providing advice and support for those affected by asbestos related diseases. All the participants felt well informed about the legal aspects related to this condition and how to claim compensation.

Anita describes not wanting to hear about making claims for compensation during the bad news consultation. She felt that claiming was the last thing on her mind following the shock of her diagnosis:
Anita:
But that was the last thing on my mind. I can't be bothered to Interviewer: go through claiming you know it was such a shock ahm.
$\begin{array}{ll}\text { Anita: } & \text { So ahm I don't think } \\ & \text { early on in a way. }\end{array}$ Of course.
Interviewer: It might be better for that to come later
Anita: $\quad$ Just to leave it a bit so you get over your diagnosis and get settled down a bit and then may be say you can apply for compensation.
Interviewer: Because everything is so overwhelming.
Anita: It is yes it is such a shock really yeah.

The issue about the timing of information related to claiming compensation is found found in other studies [4]. The best time to introduce information about financial claims is not clear and raising the issue at diagnosis can be a distraction from the emotionally charged news of an incurable disease.

Few of the participants had received a palliative care referral. Anita had asked for a palliative care referral, she had recently received a home visit from the palliative care nurse which she found very helpful and was now referred to a hospice breathlessness clinic. Gill had also been referred to palliative care but had yet to receive a home visit. None of the other participants report a palliative care referral. 


\section{Discussion}

The participants in this study struggled to come to terms with their illness during this early phase and experienced the shattering of their hopes and dreams for the future. Uncertainty, has been found to be related to emotional distress and with lower levels of satisfaction with life [27]. Psychosocial distress is reported as a serious issue for patients and families living with mesothelioma [4,10,11]. Participants in this study describe their own short-term perspective on coping with the illness such as burying one's head in the sand, denial and avoiding negative information. This enables some participants to keep some control of the situation and helps with coping in the early stage of the illness. However the longer term perspective was on the participants' mind and they were worried and anxious about how they would physically deteriorate and when this would happen.

In a study of patients with small cell lung cancer [18] it was noted how patients use narratives of restitution and optimism about their illness and treatment early on in the illness. Regarding the patients in the present study narratives of restitution were largely absent. Restitution narratives were unavailable to participants because everything they hear and read about the disease is so negative. All the 'bad news' around the illness compounded by the reported non-verbal and verbal communication from health professionals can't sustain narratives of restitution [4,11]. Frank [28] describes illness as a loss of predictability of the functions of the body and involves learning to live with a loss of control [28]. There is evidence in this study of a significant symptom burden alongside the psychosocial distress caused by living with uncertainty. Coupled with breathlessness on exertion is the feeling of fatigue and tiredness which further restricts participants physical capacity. Some patients coped well with physical restrictions and others struggled to adapt their lifestyle and pace their activities. Some people adapt to the situation involving chronic illness more easily while for others there is a 'crisis of control' [28]. Many participants in the present study suffered from day/night sweats, which caused immense distress. This symptom is not included in quality of life questionnaires for studying mesothelioma [4]. Therefore the frequency of this symptom is probably underestimated in many studies. At this early stage in the illness participants did not describe suffering from intractable pain. Symptoms and emotional suffering are linked because personal identity is shaped through the body [28]. Failure to control the body in relation to the experience of symptoms causes uncertainty and a sense of unpredictability [29]. Interventions that focus on helping patients to manage the physical and psychosocial 
aspects of their condition during the first three months will improve patient's quality of life and personal efficacy and lead to a reduction in emotional distress.

The uncertainty and lack of control experienced by participants extended to having difficulty understanding who was in charge of their care when they moved across hospitals for investigations and treatment. Chaos feeds on the sense that no one is in control [28]. Problems are reported when patients are under the care of several hospitals so that some were bewildered by the number of doctors involved in their care and could not determine who was in overall charge. This lack of continuity with medical staff results in high levels of uncertainty [27]. Participants in the present study did not have any insight into the multidisciplinary nature of the caring team and some participants were unsure of the next step in the treatment trajectory which engendered feelings of insecurity and led to a lack of control and ability to plan.

Clinicians delivering the diagnosis of MPM need to carefully consider the best and most sensitive way to deliver the 'bad news' and the timing of information about claims for compensation. There is evidence that this should not come in the diagnostic consultation. Few patients had a palliative care referral and the reason for this is unclear. Wide variations in palliative care referrals are reported in other studies leading to inadequate symptom relief and emotional care [7].

Patients need the opportunity of a home visit with a specialist nurse/palliative care practitioner soon after the diagnosis and this should occur within the first three months of the disease. This visit should enable the patient to tell their story of this illness often characterised by contact with asbestos and enable the proactive assessment and management of psychosocial distress and symptoms [30]. Patients should be given the opportunity to voice their worries and concerns particularly about the risk of passing the disease on to family members and a plan should be put in place for management and referral strategies for specialist psychosocial support should it be deemed necessary in the short and longer-term.

\section{Conclusion}

The results of this study identify the emotional turmoil experienced by patients during the first three months of the illness. Uncertainty and loss of control were key features of the patient experience causing immense distress. Uncertainty around how they 
would decline led some patients to avoid information and bad news, by burying their head in the sand. Palliative care referrals were unusual during the first 3 months of the disease. This situation seems to relate to the short-term perspective taken by the health care professionals during the first three months of the illness. Patients had to find their own strategies and resources to cope with the illness at this early stage.

Limitations of the study

This study is limited by a small sample size and participants were living in an affluent part of the UK who were interviewed during the first three months following diagnosis. Therefore the findings of this study may not be generalisable to the experience of patients in other phases of the illness trajectory and in other parts of the UK.

Funding: Surrey, West Sussex and Hampshire Cancer Network generously funded this study. I have no financial relationship with the organisation that funded the study.

\section{References}

1.Yates DH, Corrin B, Stidolph PN, Browne K. Malignant mesothelioma in south east England: clinicopathological experience of 272 cases. Thorax 1997; 52: 507-512.

2. O'Brien MER, Watkins D, Ryan C, Priest K, Corgbishley C, Norton Ashley S, Rowell N, Sayer R. A randomised trial in malignant mesothelioma (M) of early (E) versus delayed (D) chemotherapy in symptomatically stable patients: the MED trial. Annals of Oncology 2006; 17: 270-275

3. Peto J, Hodgson JT, Matthews FE, Jones JR. Continuing increase in mesothelioma mortality in Britain. The Lancet 1995; 345:535-539. 
4. Clayson $\mathrm{H}$. The Experience of Mesothelioma in Northern England. Unpublished MD thesis. Academic Unit of Supportive Care: University of Sheffield, UK 2007.

5. Hughes N. The first time I told my story. A phenomenological study of the lived experience of mesothelioma. Unpublished Msc Dissertation, University of Surrey: Guildford, UK 2006.

6. Lee S, O'Connor M, Chapman Y, Humphreys J, Hamilton V, Cass B. A Very Public Death. Dying of mesothelioma and asbestos-related lung cancer in the Latrobe Valley. Faculty of Medicine, Nursing and Health Sciences, Monash University, Frankston, Victoria 3199, Australia 2008.

7. Hawley R, Monk A, Wiltshire J. The Mesothelioma Journey: Developing strategies to meet the needs of people with mesothelioma their family carers and health professionals involved in their care. Sydney:The University of Sydney, Australia 2004.

8. Moore S, Darlison L, Todd AM. Living with mesothelioma. A literature review. European Journal of Cancer Care 2010; 19:458-468

9. Hollen PJ, Gralla RJ, Liepa AM, Symanowski JT, Rusthoven JJ. Adapting the lung cancer symptom scale (LCSS) to mesothelioma. Cancer 2004; 101:587-595.

10. British Lung Foundation Survey of mesothelioma patients and their carers. London: British Lung Foundation, 2009.

11. Sweeney K, Toy L, Cornwell J. A Patient's Journey Mesothelioma. BMJ 2009; 339:511-514.

12. Charmaz C. Good Days Bad Days The self in chronic illness and time. Rutgers University Press: New Jersey, 1997.

13. Lebovits A, Strain J. Psychiatric correlates of asbestos exposure. American Psychiatric Ass. 1984.NR85

14. Lebovits A. Industrially Acquired Pulmonary Disease. Advances Psychosomatic Medicine 1985; 14:78-92.

15. Ehrenreich B. Smile or die. How positive thinking fooled America and the world. London: Granta Publications, 2009.

16. Willig C. Cancer diagnosis as discursive capture: Phenomenological repercussions of being positioned within dominant constructions of cancer. Social Science and Medicine 2011; 73: 897-903. 
17. Charmaz C. Loss of self; a fundamental form of suffering in the chronically ill. Sociology of Health and IIIness 1983; 5:168-195.

18. The A-M. Palliative care and communication. Buckingham: Open University Press, 2002.

19. Costain Schou K. Awareness contexts and the construction of dying in the cancer treatment setting: 'micro' and 'macro' levels in narrative analysis, in I Clark The Sociology of Death (pp. 238-63) Oxford: Blackwell Publishers, 1993.

20. Pardon K, Deschepper R, Vander Stichele R, Bernheim JL et al Changing preferences for information and participation in the last phase of life: a longitudinal study among newly diagnosed advanced lung cancer patients. Support Care Cancer 2012 (In press).

21. Glaser B, Strauss A. The discovery of grounded theory Chicago: Aldine, 1967.

22. Charmaz C. Constructing grounded theory: A practical guide through qualitative analysis. London: Sage, 2006.

23. Seale C. The quality of qualitative Research, London, Sage 1999.

24. Strauss AL, Corbin J. Grounded theory methodology and overview In NK, Denzin, YS Lincoln, Strategies of qualitative inquiry (pp.158-183) Thousand Oaks: Sage, 1998.

25. Koocher GP. Terminal care and survivorship in paediatric chronic illness. Paediatric Health Psychology 1984; 4: 571-583.

26. Westbrook MT, Viney LL. Psychological reactions to the onset of chronic disease. Social Science and Medicine 1982; 16:899-905.

27. Mishel MH. Living with chronic illness: Living with uncertainty. In Funk SG, Tornquist EM., Champagne M.T, Wiese RA Key Aspects of Caring for the Chronically III. New York, Springer 1993: 47-58.

28. Frank A.W. The wounded story teller. The University of Chicago Press, Chicago 1995.

29. Beech N, Arber A, Faithfull S. Restoring a sense of wellness following colorectal cancer: a grounded theory. Journal of Advanced Nursing 2012; 68:1134-1144.

30. Hughes N, Arber A. The lived experience of patients with pleural mesothelioma. International Journal of Palliative Nursing 2008;14: 66-71. 